\title{
Governance of global health research consortia: Sharing sovereignty and resources within Future Health Systems
}

\begin{abstract}
Global health research partnerships are increasingly taking the form of consortia that conduct programs of research in low and middle-income countries (LMICs). An ethical framework has been developed that describes how the governance of consortia comprised of institutions from high-income countries and LMICs should be structured to promote health equity. It encompasses initial guidance for sharing sovereignty in consortia decision-making and sharing consortia resources. This paper describes a first effort to examine whether and how consortia can uphold that guidance. Case study research was undertaken with the Future Health Systems consortium, which is funded by the UK Department for International Development and performs research to improve health service delivery for the poor in Bangladesh, China, India, and Uganda. Data were thematically analysed and revealed that proposed ethical requirements for sharing sovereignty and sharing resources are largely upheld by Future Health Systems. Facilitating factors included having a decentralised governance model, LMIC partners with good research capacity, and firm budgets. Higher labour costs in the US and UK and the Department for International Development's policy of allocating funds to consortia on a reimbursement basis prevented full alignment with guidance on sharing resources. The lessons described in this paper can assist other consortia to more systematically link their governance policy and practice to the promotion of health equity.
\end{abstract}

Key Words: ethics, governance, justice, equity, research consortia, global health, health systems research 


\section{Introduction}

Global health research partnerships are increasingly taking the form of consortia that conduct programs of research and research capacity strengthening in low and middle-income countries (LMICs). These consortia are alliances of universities, research institutes, and other organisations such as commercial companies (Dockrell 2010). Their research programs focus on product development (vaccines, drugs, diagnostics) for specific diseases; the delivery, safety, and costeffectiveness of particular medical products for specific diseases; broader aspects of health systems (governance, financing, service delivery, human resources, information technology); or the social or structural determinants of particular diseases (Anonymous).

Such partnerships have increasingly been recognised as a "powerful tool" to reduce global disparities in health and research capacity (CHRD 1990, xvii; Ministerial Summit on Health Research 2004; Global Ministerial Forum on Research for Health 2008). By bringing together numerous partners that may span several sectors, consortia can leverage their aggregate intellect and resources to promote health equity. They are seen as an effective way to reduce duplication in research and develop research capacity, including by building closer South-South linkages (Dockrell 2010).

Governance has been identified as a key mechanism for establishing and attaining health equity objectives (Ruger 2011; Ruger 2012; Gostin 2014). Governance of research consortia refers to their decision-making, encompassing the processes by which consortia make decisions about their goals, priorities, and allocation of resources; who makes such decisions; and the outputs of these processes. An account from political philosophy called shared health governance has been applied to derive initial ethical guidance on what features of governance are necessary for consortia (comprised of institutions from high-income countries and LMICs) to help reduce global health disparities (Anonymous). Shared health governance describes the type of governance needed to achieve global health justice and identifies five components as essential—advancing the goals of health justice, shared sovereignty, shared resources, shared responsibility, and mutual collective accountability (Ruger 2011; Ruger 2012). The proposed ethical framework details what consortia should do to uphold each of the components of shared health governance (Anonymous). It was developed by 
bioethicists from the US. Feedback and input on its content have been sought from LMIC bioethicists and researchers.

According to the ethical framework, advancing health justice means three elements of consortia's research enterprise are key—-their research priorities, capacity development, and promotion of research uptake and translation—because these elements can link their activities to the ends of justice $^{1}$ (Anonymous). Global health research consortia should ensure such elements are present and structured to promote the health of those with the poorest health globally. Sharing sovereignty calls for consortia to undertake priority-setting in an inclusive and deliberative manner led by LMIC partners. Sharing resources requires that consortia partners receive their fair share of consortia resources and allocate them to equity-oriented research, research capacity strengthening, and research translation activities. Mutual collective accountability entails agreement by consortia members on both the goals of their joint work and how it's to be judged (Anonymous). More detail on how consortia should uphold each component of shared health governance is provided in Figure 1.

Although ethical guidance linking governance of transnational global health research consortia to the promotion of health equity is starting to be defined, practices capable of fulfilling it have yet to be described. Some work in bioethics has assessed whether global health research collaborations generate social value and advance health justice in LMICs (Anonymous et al. 2014; Lairumbi et al. 2008), but these studies do not explicitly focus on collaborations' governance. As part of a first effort to explore whether and how consortia uphold shared health governance, case study research was undertaken with the Future Health Systems (FHS) consortium. FHS is a health systems research consortium funded by the UK's Department for International Development (DFID).

This paper examines the achievement of shared sovereignty and shared resources by FHS. These components of governance are especially challenging to realise in contexts of power disparities, so it is vital to document existing models of practice that promote their fulfilment. (FHS governance was also examined for furthering the ideals of health justice, but those findings are reported in a separate

\footnotetext{
${ }^{1}$ Here, it is acknowledged that philosophers have conceptualised health justice in various ways and it continues to be a matter of debate (see Shue, 1996; Powers and Faden, 2006; Daniels, 2008; Ruger 2012).
} 
paper.) Data collected through in-depth interviews, observation at a 2014 FHS annual meeting, and examination of FHS documents were thematically analysed for alignment with proposed framework requirements for shared sovereignty and shared resources. Ultimately, the paper provides useful lessons for global health research consortia seeking to more systematically link their governance to their equity objectives. Insights from this case study also helped identify areas for expansion of the ethical framework.

\section{Proposed ethical guidance for sharing sovereignty and resources}

Before assessing whether and how FHS achieved shared sovereignty and shared resources, it is necessary to first provide additional details on what doing so entails. Since shared sovereignty requires inclusive and deliberative decision-making (Ruger 2011), the ethical framework calls for consortia priority-setting processes to have both features. Inclusion encompasses not only who is invited to be present for priority-setting but also how they are involved in the process (Young 2000). According to the framework, being inclusive means participants in priority-setting represent a wide spectrum of roles (senior researchers, junior researchers, research implementers) and demographics (gender, institutions) within a consortium. Similar numbers of participants with these roles and demographic characteristics should be achieved (Anonymous). Certain roles, e.g. senior researchers, and demographics, e.g. men, should not be disproportionately present relative to one another in order to ensure that certain groups do not dominate priority-setting by force of numbers. It is highly desirable for priority-setting processes to be informed by research users and beneficiaries within consortia partners' countries, e.g. health policymakers, providers, and patients, especially those from disadvantaged groups (Anonymous). Additionally, measures should be in place to reduce the impact of power disparities on participants' opportunity to voice their ideas for priorities such as those between participants from different LMICs, researchers and implementers, and senior and junior researchers. This ensures that the views expressed in priority-setting processes are not solely those of more powerful actors.

Where countries are unable to ensure their population's health, global actors have an obligation of justice to take on a "supportive and facilitative role" to assist them to meet their responsibility and to 
build their capacity to do so on their own (Ruger 2008, 433). The ethical framework, therefore, holds that when high-income country consortia members assume a role in priority-setting, it should at most consist of helping LMIC consortia members identify and decide upon research priorities that are relevant to their countries. It is then highly desirable that priority-setting processes involve an equivalent or greater mass of participants from LMICs and be structured to ensure that they have an equal (or greater) chance to share their proposed research priorities relative to participants from highincome countries (Anonymous).

According to the framework, being deliberative means participants are able to voice their ideas for consortia research priorities and justify them to the entire group, which should then have an opportunity for in-depth discussion, where the pros and cons of various proposals are debated. Proposals are refined and/or weeded out and participants coalesce around their preferred option(s). The final consensus should reflect the joint intentions of participants rather than the aggregation of unchanged individual preferences or simple agreement to the preferences of certain participants who have power over other participants (Anonymous). Consensus as joint intentions means that consortia members have modified their positions in light of the positions of others during the deliberative process. Deliberation forges novel purposes/proposals that reflect the ideas of the group as a whole as opposed to the unchanged views of powerful participants within it (Richardson 2002).

Sharing resources means that partners receive their fair share of resources and make efficient use of them to promote health equity (Ruger 2011). As such, the framework proposes a greater proportion of high-income country partners' resources go to assisting with LMIC partners' research than to supporting their own research in LMICs. Here, LMIC partners' research is distinguished from highincome country partners' research based on who leads the project and whose interests are primarily served by its conduct. High-income country partners should generally not be allocated more total consortia resources than LMIC partners, though exceptions may exist (e.g. research involving highly expensive processes like genome sequencing that are largely performed in high-income countries). This principle applies to the overall consortia budget, irrespective of existing cost structures for labour and travel. The amount of resources allocated to LMIC partners should correspond to their needs in terms of achieving their research agenda, the research capacity strengthening they identify as being 
required, and their research translation objectives. As a result, it is acceptable for LMIC partners to receive somewhat (but not significantly) unequal amounts of consortia resources. All consortia partners should have direct control over the resources allocated to them each year (Anonymous).

\section{Methods}

\section{The case under study}

FHS was selected as our case study because it focuses on generating knowledge to improve service delivery for poor populations in LMICs. This made it likely that FHS would provide useful information on whether and how ethical guidance linking consortia governance to the promotion of health equity could be upheld. FHS consists of six partners: Johns Hopkins Bloomberg School of Public Health (JHSPH) (USA), the Institute of Development Studies (IDS) (UK), Makerere University School of Public Health (Uganda), the International Centre for Diarrhoeal Disease Research, Bangladesh (ICDDR,B), the Indian Institute of Health Management Research (IIHMR), and the China National Health Development Research Center (CNHDRC). The consortium is now operating in its second phase, which is slated to run from 2011 to 2016. (Phase-1 ran from 2005 to 2010.) This included a year-long Inception Phase in 2011, where the consortium's research questions and designs were further developed and refined. Implementation of research projects commenced in 2012 in partner LMICs.

$\mathrm{JHSPH}$ is leading the management of FHS and a JHSPH faculty member is its CEO. FHS also has two research directors, one from JHSPH and one from ICDDR,B. It convenes a Consortium Steering Committee that guides the overall direction of the consortium and consists of nine members: the principal investigator from each partner institution, the consortium CEO, its research manager, and its research uptake manager.

\section{Case study research methods}


This case study focused on FHS Phase- 2 because it was ongoing at the time of data collection. Data were gathered between June and December 2014 using a triangulation approach that relied on indepth interviews, document analysis, and, to a lesser extent, observation (because FHS prioritysetting and resource allocation had largely been completed). Fifteen semi-structured in-depth interviews were conducted by LB [initials changed for blinded review] with: steering committee members from high-income countries (four interviews), steering committee members from LMICs (four interviews), and junior researchers from LMICs (seven interviews). Junior researchers were selected based on having participated from the outset of FHS Phase-2 and spanning the different LMIC partners. Six women and nine men were interviewed. A series of open-ended questions were used in interviews that included being asked to describe who was responsible for deciding the consortium's Phase-2 research priorities, who were consulted as part of the process, and who made decisions about the allocation of FHS resources. Junior researchers were asked additional questions about their inclusion in priority-setting such as whether they were given an opportunity to provide input in the selection of FHS Phase-2 research priorities, when they were consulted (pre-Inception, Inception, or post-Inception phase), and whether they thought they had an equal opportunity to provide input relative to senior researchers.

Interview data were supplemented by an examination of FHS documents and by observation at the 2014 FHS annual meeting. Observation primarily served to build knowledge of FHS members and decision-making processes at annual meetings in order to facilitate more accurate interpretation of interview data. Examined documents included: Phase-2 annual meeting agendas and minutes; annual reports; budgets; and DFID Research Programme Consortia Terms of Reference (2009). Annual meeting attendance was analysed in terms of who was present and how they participated (Table 1). For example, the amount of discussion time and small group work served as an indirect measure of opportunity for deliberation.

Interviews were transcribed verbatim and thematic analysis was undertaken by two coders in the following five phases: initial coding framework creation, coding, inter-coder reliability and agreement assessment, coding framework modification, and final coding of entire dataset (Hruschka et al., 2004; Campbell et al. 2013). The categories and sub-categories in the initial coding framework were derived 
inductively and deductively, relying on both shared health governance concepts and transcripts' content. Using the initial coding framework, the two coders undertook an iterative process of coding a transcript, assessing inter-coder reliability and agreement, and modifying the coding framework (Hruschka et al., 2004). Given the high levels of inter-coder agreement (100\% and $98 \%$ at the primary and secondary levels respectively) achieved after two iterations of coding, the lead coder then employed the revised coding framework (and NVivo Version 10) to re-code all fifteen transcripts, which is consistent with Campbell et al. (2013).

Once categories were identified that pertained to shared sovereignty and shared resources, their collated data extracts were assessed to determine whether they provided evidence that FHS upheld the proposed ethical guidance. This type of analysis has been previously applied in Anonymous et al. (2014). To ensure an accurate interpretation of the data, interviewees from five FHS partners provided feedback on an early draft of the manuscript.

Ethical approval for the study was obtained from [removed for blind review] Institutional Review Board, which deemed the study exempt from review.

\section{The authors' positionality}

The authors are members of FHS, performing ethics research relevant to the consortium, and FHS funding partially supported this case study. [Removed for blind review] was not a member of FHS during priority-setting, joining just prior to conducting the case study. The potential for the authors' role in FHS and their being from a high-income country to affect data analysis is acknowledged. Given this, reflexivity in the thematic analysis process included constant questioning of whether consideration of FHS priority-setting and resource allocation was affected by the authors' positionality. To further promote validity and reliability of data analysis, thematic analysis was undertaken by two coders, one of whom was not part of FHS (and is not a co-author of the paper).

\section{Results}




\section{Shared sovereignty}

Interviewees described two levels of priority-setting being undertaken by FHS—at the consortiumlevel and at the country-level. At the country-level, FHS LMIC partners each undertook separate processes to identify their research priorities. At the consortium level, priority-setting resulted in the selection of three "cross-cutting themes" that apply across FHS. Country-level priority-setting drove consortium-wide priority-setting in FHS, with country-level research priorities comprising a key consideration in the selection of Phase-2 cross-cutting themes. The function of the cross-cutting themes was primarily to establish a "space" for LMIC partners to communicate with and learn from one another about their largely diverse research projects. They could discuss their research in the context of shared themes. The cross-cutting themes also functioned as a way for the consortium to bring country-specific findings together in publications in a more "generalizable" form. The "push" for cross-cutting themes came from DFID, which required greater coherence of research priorities across FHS Phase-2.

FHS priority-setting processes at the country and consortium levels will subsequently be described and assessed for alignment with the proposed ethical framework. Importantly, FHS priority-setting draws attention to the fact that consortia can adopt a decentralised and bottom-up model of operations, a centralised and top-down model of operations, or a model comprised of both top-down and bottom-up elements, with FHS choosing the former. At present, the ethical framework does not distinguish between centralised and decentralised consortia in its guidance. This matter will be revisited in the paper's discussion.

Country-level priority-setting

Junior and senior researchers from all FHS partners affirmed that LMIC research teams were primarily responsible for selecting their respective Phase-2 research priorities. According to a LMIC researcher, "you had a lot of freedom really in choosing what is relevant for the country. So in that sense, I mean, it's the consortium, but it's not a regimental, top-down consortium." For some research 
teams, this autonomy meant the entire research team collectively selected their research priorities whereas, for others, multiple senior researchers shared the decision-making role.

LMIC researchers described their research priorities being first developed by their teams, with some degree of consultation with research users and beneficiaries from their country. This consultation was intended to ensure country-level priorities reflected the on-the-ground reality. All LMIC research teams spoke with national policymakers from their ministries of health and/or sub-national health officials in the districts/states in which they performed their research. In Bangladesh, community members from the sub-district in which the ICDDR,B team does research were also consulted. In India, the IIHMR team "did not talk to all common people, but the civil society [organisations] can be taken as a representative of the residents."

These LMIC stakeholders typically did not share decision-making on research priorities with FHS research teams. However, priority-setting was often driven by their demands. Two junior LMIC researchers discussed how this unfolded in their countries:

"There was a program going on in the health and family welfare department, Government of West Bengal. Its name was Health System Development Initiative. The commissioner of that program... requested that if you really want to do something in West Bengal please give us some idea what's the health status and what we need to do in the context of Sundarbans... it was the direct request from the government people."

"We always talk with the community with the - with participant of our research on what their demand is. And we found the issue from their perspective. We are not telling... This is from their opinion that it will be better if you are linking the village doctor with the formal doctors." As demonstrated by these examples, the type of stakeholder whose demands were influential in country-level priority-setting varied by LMIC partner. The focus of the resultant priorities, nonetheless, was to address inequalities in access to health services by disadvantaged populations (i.e. the Sundarbans' population in India and the population of a rural sub-district in Bangladesh). 
Once country-level priorities were formulated, feedback was sought from the wider FHS membership. When FHS members offered feedback, a LMIC researcher noted "[i]t's more helping people with more input and... other members, like Johns Hopkins and IDS, that play supportive role here. It is really uncommon that somebody will say, 'No, no, no. You don't do that,' that kind of thing." Here, the interviewee's statement casts high-income country partners as playing a "supportive" role in countrylevel priority-setting. This was further affirmed by another junior LMIC researcher, who reported that "the mentoring is always there for-I mean, for the betterment of the research, not in a putting something on the countries that, "You should do that in your area." LMIC research teams could take or leave the feedback they received. They were responsible for setting their final research priorities and revisiting them as needed throughout Phase-2. A variety of approaches (pre-experimental, quasiexperimental, participatory action) and methods (cross-sectional survey, social network analysis, case study, and Geographic Information System mapping) were utilised in LMIC partners' research, with all partners employing both quantitative and qualitative methods.

\section{Alignment with ethical guidance}

FHS priority-setting processes at the country-level were largely consistent with ethical guidance on shared sovereignty. LMIC researchers' mode of inclusion was "decision-making" whereas highincome country researchers' mode of inclusion was "consultation" and "facilitation". The range of roles and demographics within FHS LMIC research teams were for the most part represented in countrylevel priority-setting because, with the exception of CNHDRC, all team members were part of the processes. However, interview data does not speak to whether research implementers were included or whether qualitative equality within LMIC research teams was promoted, e.g. whether processes were structured to promote senior and junior researchers or male and female researchers having an equal chance to voice their perspectives.

LMIC stakeholders were consulted by all FHS LMIC research teams as per framework guidance. The research user perspective was acquired from national and sub-national policymakers. The research beneficiary perspective was drawn from civil society organisations and members of the research population. Even so, one area where some LMIC research teams may have had room for 
improvement was with respect to engaging disadvantaged populations (i.e. those with the worst health) within their countries. Such stakeholders were not explicitly mentioned by interviewees, though such engagement might have occurred in FHS Phase-1. A lack of engagement may be problematic because policymakers (especially at the national level) might not have a robust understanding of how the health system is failing disadvantaged populations within their country. It is, therefore, vital that members of disadvantaged populations or, at a minimum, civil society organisations legitimately representing them be consulted during priority-setting in order for researchers to obtain a better picture of health system shortcomings related to such populations' access to services and financial protection. If such engagement did occur in FHS Phase-1, it, nonetheless, raises questions about when engagement with disadvantaged populations should occur in long-term research partnerships. Is it ethically permissible to only engage such populations during priority-setting in a consortium's first phase of research? Or must they be continually engaged in each round of priority-setting? Their depth of participation would seemingly be greater in the latter option.

FHS-wide priority-setting

Senior and junior researchers from high-income countries and LMICs affirmed the Phase- 2 crosscutting themes were first proposed by researchers from JHSPH and IDS. These researchers bore certain considerations in mind when developing the cross-cutting themes, including the funding call to which they intended to apply (DFID's Research Programme Consortia) and relevance to multiple LMIC partners' Phase-2 research interests. As noted by one interviewee, “as you're probably aware, FHS has been quite decentralized and quite, sort of, let 1,000 flowers bloom, so in a way, it wasn't like ruling out what we were not going to do; it was more about thinking about how this diversity of things that we were interested in could be presented in a coherent fashion."

After senior researchers from JHSPH and IDS proposed the cross-cutting themes, they were discussed and fleshed out by the entire FHS steering committee at a 2009 meeting in Amsterdam, which was held prior to submitting the Phase-2 grant proposal to DFID. Junior researchers were not 
included at that meeting. At the time, the steering committee composition was $44 \%$ LMIC members and heavily skewed towards men (Table 1 ).

Following the Amsterdam meeting, input on the cross-cutting themes was then sought from the wider consortium membership. Here, inclusion was heavily dependent on attendance at FHS annual/biannual meetings, though some teams may have consulted with junior researchers who did not attend prior to these meetings. Junior researchers from LMICs affirmed the "[s]election of the themes was mainly done during some FHS workshops. I didn't participate but I know some of our colleagues, especially the PIs and some of the other team members attended this meeting... and for us later on we just saw what things they had selected."

Over a series of meetings between 2010 and 2011, the Phase-2 cross-cutting themes were discussed and refined by representatives of the wider consortium (Table 1). All meetings were attended by nearly equal numbers of individuals from high-income countries and LMICs, though fewer individuals from LMICs typically attended and two partners (JHSPH and Makerere University) were consistently overrepresented. Attendance by LMIC consortium members was facilitated by holding meetings in LMICs or after international conferences. The meetings were also generally characterised by less representation of junior researchers and women. (This lack of female representation may reflect that overall consortium membership favoured men, which, in turn, may be due to there being fewer female (senior) health systems researchers at FHS partner institutions. ${ }^{2}$ )

Meeting agenda analysis and interviewees indicated that opportunities existed for individuals to share their ideas regarding the consortium's cross-cutting themes. The Montreux meeting, for example, included four hours of discussion time on the cross-cutting themes and the Brighton meeting included six hours of small group work on the cross-cutting themes. According to a junior LMIC researcher, "in fact, we were teased with those themes before travelling to Brighton and then in Brighton we had an extensive discussion on each of the themes. It was not like a given one like 'well, this is

\footnotetext{
${ }^{2}$ Even if this is true of FHS partner institutions, it was not necessarily true of FHS research teams from those institutions, with one LMIC interviewee reporting a gender balance across his research team.
} 
going to happen and you guys have to work on it.' We had smaller group discussions and stuff like that.

Annual meetings in FHS Phase-2 were purposefully structured to promote qualitative equality amongst senior and junior researchers. According to an interviewee,

"when [steering committee member] came into FHS...All of the country team leads were men, and in the [annual meetings], it was mainly the country team leaders having this quite sophisticated dialogue between themselves, and it seemed like everyone else sort of sat and listened and learned. So [steering committee member] wanted to change that and make it more participatory... So we've done posters, sometimes, where everyone does a poster and then stands in front of their poster and talk about it. Small group work where you split up in different ways and come back and report - and just by breaking it out, I think it's given more of a voice to people who might not be able to stand up to [names of steering committee members] in a big room and say, "I disagree with you. I think $x, y$, or zed," but who could do it in a group." The addition of female researchers in senior positions and/or with strong voices also served to diminish the dominance of senior male researchers' voices at consortium meetings in Phase-2.

Aside from encompassing deliberation, FHS-wide priority-setting was described as having features of negotiation between powerful and less powerful participants. It was not simply a process of selecting the themes that best encapsulated FHS country-level research but also a process of negotiation between "interest groups", where themes were selected on the basis of how hard groups were willing to "push" for them. This characterisation applied to the theme of complex adaptive systems (CAS), which refers to undertaking health systems research using CAS theories and methods (to some degree) when designing and conducting studies. According to one interviewee, "interest groups" could represent different methodological interests in health systems research, a result of the field being so multidisciplinary. Power dynamics between interest groups could stem from their members representing high-income country partners, LMIC partners, or different LMIC partners. Adoption of the CAS theme, for example, was "pushed" by a group that included senior high-income country researchers, leading to perceived tensions with certain senior LMIC researchers. However, this did not necessarily mean these LMIC researchers automatically acceded. As noted by one interviewee, 
"[s]o it was actually quite a strong pushback. It was interesting because it was very much a, you know, [a high-income country partner] saying, 'This is very much what we would like to do,' and [two LMIC] partners turning around and saying, 'Nope. No can do."' The matter was revisited by the consortium as a whole at the Kampala meeting in 2012. This spurred deliberative dialogue with features of negotiation:

"So we came back at it again in [the annual meeting], but softly, softly. We were much moreless pushing - you know, we hadn't anticipated such a reaction... our strategy was that we would provide staff time to help support that, and I think also, we thought that by providing slightly more junior staff to take that forward, it would seem less of a power play than [name of senior high-income country researchers] walking in, saying, 'We'd like you to do this."'

Ultimately, through a process of deliberation and negotiation, nine interviewees, including senior and junior researchers from high-income countries and LMICs, affirmed genuine consensus was reached on the cross-cutting themes, including CAS. In such processes, power dynamics can affect what consensus looks like in terms of its content (what priorities are agreed upon) and the type/nature of agreement (joint intentions, aggregation of individual preferences, simple agreement). The push for the CAS theme eventually resulted in its adoption, but LMIC interviewees did not articulate feeling powerless or only accepting it because they felt they had no choice. .

\section{Alignment with ethical guidance}

Consistent with the ethical framework, participants in FHS-wide priority-setting represented the range of partner institutions, senior and junior researchers, and both genders. However, research implementers were not involved and the mass of LMIC participants (at annual meetings) was slightly lower than it ideally ought to be. There was overrepresentation of senior researchers and, for the most part, LMIC research users and beneficiaries were not directly involved in selecting the Phase-2 crosscutting themes.

The selection of cross-cutting themes was not led by LMIC consortia members, though they did share decision-making with high-income country consortia members. These modes of participation are 
somewhat inconsistent with the proposed ethical guidance, as high-income country consortia members' role was not limited to facilitation. In terms of promoting qualitative equality, FHS-wide priority-setting processes were at most structured to ensure that LMIC participants had an equal opportunity to voice their ideas for cross-cutting themes relative to high-income country participants. It is possible that senior researchers had greater opportunity to influence FHS-wide priority-setting because they were more likely to attend annual meetings. However, mechanisms were employed at annual meetings to promote the voices of junior researchers being raised.

\section{Resource allocation}

A greater proportion of JHSPH and IDS resources were allocated to assisting FHS LMIC partners conduct research than to their own research. Line items on the FHS Phase-2 budget included: personnel, travel, programme costs, equipment, capacity building, research uptake, and overheads. IDS and JHSPH's overall budgets primarily went to personnel costs (48.3\% and $61.9 \%$ respectively). These costs largely supported their staff's work on LMIC partners' research in Bangladesh, China, India, and Uganda. Very few FHS resources were directed towards programme costs or equipment for JHSPH or IDS research.

However, JHSPH and IDS were allocated more total resources than LMIC partners, which is inconsistent with the ethical framework. $30.1 \%$ of the total FHS Phase-2 budget went to JHSPH, $19.6 \%$ to IDS, $18.8 \%$ to Makerere University ${ }^{3}, 9.6 \%$ to ICDDR,B, $9.5 \%$ CNHDRC, and $8.2 \%$ to IIHMR (Bennett et al., 2012). This was primarily due to higher costs for overheads, personnel, and travel in high-income countries.

Interview data indicated that FHS resources were distributed to LMIC partners according to need via a process of negotiation with FHS research directors and CEO. As noted by an interviewee, "everyone [each partner] puts in a budget and we hash it out." DFID Research Programme Consortia grants have a capped budget. As a result, once all FHS partners had submitted their proposed budgets,

\footnotetext{
${ }^{3}$ Half of this money went to the FHS research team at Makerere University and the other half went to the Africa Hub, a capacity development collaboration between FHS and seven schools of public health in East Africa, including Makerere University.
} 
steering committee members had to ensure that they collectively did not surpass the total grant amount.

After the FHS Phase-2 budget was set, interviewees indicated that it was fixed and partners had control over their resources, as per framework guidance. One interviewee stated:

"What I'm saying is Hopkins ceded control over country budgets... the indicative budget turned into firm budgets, so people controlled their budgets. Therefore, the project lead did not have a lot of leverage except kind of moral suasion or - you know, but no financial power in the lead." Another interviewee affirmed:

"[W]e have a master budget that sets out funding that will go to each of the country teams throughout the entire six years of the project. So countries have a fair amount of financial security and financial predictability, which, I think, also puts them more in the driving seat. It's not like they're continually having to negotiate with [JHSPH] around their budgets."

The only area where certain (but not all) LMIC partners noted that decision-making over resources budgeted to them moved out of their control was when they had unspent resources at the end of a financial year. Rather than leaving it up to individual partners to re-allocate their unspent funds, FHS moved the locus of decision-making to the consortia-level. Discussions were held within the steering committee as to whether these partners' funds could be redistributed and then the decision of how to redistribute them was taken to the wider consortium at annual meetings. In contrast, another LMIC partner reported its unspent money was distributed to its research team over subsequent years.

The administering organisation (JHSPH) did not disburse each partner's share of FHS resources at the start of every financial year, which is inconsistent with the ethical framework. JHSPH had a contract with DFID and sub-contracts with each FHS partner, who then received their resources on a reimbursement basis. For JHSPH, this was a matter of legal policy. DFID also paid JHSPH on a reimbursement basis, which not only required $\mathrm{JHSPH}$ to advance funds to FHS partners at its own risk but also limited the capacity of LMIC partners to serve as the administering organisation. 
FHS resources were allocated to research, research capacity-building, and research translation activities. At the consortia-level, more resources were directed to research uptake $(£ 113,889)$ compared to research capacity strengthening $(£ 66,091)$ over FHS Phase-2 (Bennett et al., 2012). At the country-level, there was variation between LMIC partners in the amount of resources allocated to research capacity strengthening versus research uptake.

\section{Facilitating factors}

Factors that facilitated FHS priority-setting aligning with ethical guidance on shared sovereignty are described in Table 2. Those most frequently cited were decentralised consortia governance, LMIC partners' control of their budget allocations, and the strong research capacity of LMIC partners. Incentives created by the DFID funding scheme were cited as well. The scheme required research to be demand-led and responsive to the research priorities of LMIC partners (DFID, 2009). DFID was praised for promoting more equitable resource allocation between consortia partners, with a highincome country interviewee stating:

they're interested to see and concerned about how much of your money goes to certain institutions, so it would be unacceptable if we had 70, 80 percent of our funding going to Hopkins and IDS.

DFID checked whether the majority of resources went to high-income country partners in the consortia it funded and required "a substantial proportion (over 30\% would not be unreasonable)" of consortia budgets go to research uptake and research capacity strengthening (DFID, 2009).

\section{Obstructive factors}

Factors that obstructed FHS priority-setting aligning with the ethical guidance on shared sovereignty are described in Table 3. They include funding environment norms and the incentives created by DFID funding scheme. The main challenges to meeting framework requirements for consortia resource allocation were DFID and high-income country partners' policies, which required consortia funds to be disbursed on a reimbursement basis, and higher labour costs in the US and UK. Funding criteria also created strong incentives for JHSPH to serve as the administering organisation. 


\section{Discussion}

This paper describes a first effort to examine whether a health systems research consortium's practice is consistent with ethical guidance linking consortia governance to the promotion of health equity. That guidance details how research consortia can achieve the five components of shared health governance-advancing health justice, shared sovereignty, shared resources, mutual collective responsibility, and shared responsibility. The case study presented here explores whether and how FHS has been able to fulfil proposed ethical requirements for shared sovereignty and shared resources.

Its findings provide lessons as to what policies and practices global health research consortia might adopt and how funders might design their grant schemes to promote health equity. Achievement of LMIC-led priority-setting was strongly facilitated by FHS adoption of a decentralised model of operations, the selection of LMIC partners with good research capacity, and the trust developed over a long-term partnership (i.e. two phases of funding). Inclusion of LMIC stakeholders was facilitated by DFID's Research Programme Consortia funding scheme requiring and supporting their engagement during a yearlong Inception Phase. Even so, inclusion of disadvantaged groups in country-level priority-setting may not have been achieved by all FHS LMIC partners; DFID's emphasis was on consulting policymakers. This potentially suggests a need for funders to provide greater incentives and resources for engaging such groups and for researchers to receive (more) training on how to engage them. Inclusion in consortia-level priority-setting was promoted by holding annual meetings (funded by DFID), having (senior) female researchers with strong voices, and conducting small group work.

It is important to note that, even in a consortium committed to advancing equity, high-income country institutions received $50 \%$ of the budget. This often reflects higher costs including salaries at highincome country institutions. Assigning tasks to more LMIC researchers or less senior high-income country researchers may, therefore, allow reallocation of funds. 
The FHS case further shows how funding policies can empower or disempower LMIC institutions and researchers to address health inequities. Requiring consortia to have three or more LMIC partners and restricting the amount of resources high-income country partners could receive is empowering. Yet giving funds on an on-claim basis and having selection criteria relating to financial management track record can be disempowering. Funders, at a minimum, need to support building LMIC institutions' capacity to manage large grants and give them opportunities to gain experience in this area.

Beyond these lessons, the case study highlights a number of gaps in the ethical guidance for consortia governance that require further exploration. It draws attention to the fact that consortia can adopt decentralised and bottom-up or centralised and top-down models of operations, which raises normative questions such as: whether bottom-up, top-down priority-setting, or a combination is ethically preferable? And if so, when is it ethically preferable (always or only under certain conditions)? This also means the framework could usefully distinguish between bottom-up and topdown consortia in its guidance. The case study shows that country-level priority-setting is more influential in decentralised consortia. This may support reformulating framework requirements for consortia-wide priority-setting, where consortia are decentralised. It may be ethically permissible to have equal representation and opportunity for high-income country and LMIC consortia members to voice their ideas for consortia-wide priorities. Having a greater mass and opportunity for LMIC consortia members to share ideas during consortia-wide priority-setting is less essential because country-level priority-setting drives the consortium's research agenda. For the same reason, it may be ethically acceptable for high-income country and LMIC consortia members to share decision-making about how to package country-level research priorities into cross-cutting themes. It is less essential that high-income country consortia members' role be limited to "facilitating" consortia-wide prioritysetting in decentralised consortia. It may also be ethically permissible not to consult LMIC stakeholders during consortia-wide priority-setting, provided that they are consulted as part of country-level priority-setting. These questions bear further consideration. 
The case study suggests that consortia may face potential trade-offs between achieving two core features of equity-oriented consortia governance-namely, LMIC-led priority-setting and enhanced research capacity of LMIC partners. FHS was able to uphold framework guidance on priority-setting because its LMIC partners had relatively good research capacity. Each LMIC research team included at least one or two very experienced health systems researchers (as well as multiple less experienced researchers). Yet having stronger LMIC institutions and/or researchers in a consortium may limit opportunities for performing research capacity strengthening. The proposed ethical guidance may, therefore, need to be nuanced such that different expectations are in place for consortia that choose to favour LMIC-led priority-setting versus those that choose to favour building LMIC institutions' and researchers' capacity. Further work is needed to explore what that differing guidance might look like.

Additionally, guidance on the engagement of LMIC stakeholders and fair negotiation (in contexts of power disparities) in consortia priority-setting demands more development. Interviewees' descriptions of priority-setting suggested that certain types of LMIC stakeholders had more influence over the selection of their research priorities than others. This draws attention to the fact that when multiple LMIC stakeholders are consulted, even where they have an equal opportunity to share their ideas, if their suggestions are different, priorities will ultimately be selected that favour the ideas of some LMIC stakeholders over others. This raises the question: whose input should researchers give more weight when selecting priorities? Should it be, for instance, national policymakers, sub-national policymakers, or disadvantaged populations?

There were a number of limitations to the FHS case study that should be taken into account. First, only one interviewee was from the CNHDRC team in China, meaning the data reported is less informed and reflective of their perspectives. Second, due to the timing of the case study research, FHS priority-setting and resource allocation processes largely could not be observed. Third, the authors' positionality as high-income country researchers may have constrained their ability to pick up certain nuances or aspects of LMIC partners' experience of FHS governance when analysing the data. Early drafts of the paper were, therefore, shared with three LMIC interviewees to get their feedback and their comments were incorporated into subsequent drafts. Fourth, by focusing on FHS Phase-2, the case study may not have fully identified how being a long-term consortium facilitates 
shared health governance, beyond that the trust developed in FHS Phase-1 promoted shared sovereignty in Phase-2. It also did not capture FHS Phase-1 priority-setting processes in great detail, which consortium members noted were different to Phase-2 processes. Future research could explore the similarities and differences between priority-setting in FHS Phase-1 and 2 in terms of shared sovereignty. Fifth, the case study may not have fully captured the non-linear process of selecting the cross-cutting themes-namely, the changes and evolution in their meanings that occurred due to their being revisited annually. Finally, given FHS is a decentralised consortium, the case study could have benefited from greater emphasis of data collection on country-level priority-setting. Future research on decentralised consortia might focus more deeply on priority-setting at the country-level relative to the consortia-level.

Ultimately, the lessons described in this paper can assist other consortia to more systematically link their governance policy and practice to the promotion of health equity. The approach described in this paper may be used by consortia wanting to evaluate their governance practice. It can also usefully be employed in future research to explore whether and how centralised consortia and/or consortia whose LMIC partners have weaker research capacity might uphold the ethical framework.

\section{References}

Bennett, S., Lewy, D., Knezovich, J., Bhuiya, A., and Peters, D. (2012). Annual report: 1 October 2011 - 30 September 2012. Baltimore, USA: Future Health Systems.

Campbell, J.L., Quincy, C., Osserman, J., and Pedersen, O.K. (2013). Coding in-depth semistructured interview: Problems of unitization and intercoder reliability and agreement. Sociological Methods \& Research, 42(3), 294-320.

Commission on Health Research for Development. (1990). Health research: Essential link to equity in development. Oxford: Commission on Health Research for Development. 
Daniels, N. (2008). Just health: Meeting health needs fairly. Cambridge: Cambridge University Press.

Department for International Development (DFID). (2009). Research programme consortia: Terms of reference. London: DFID.

Dockrell, H.M. (2010). Presidential address: The role of research networks in tackling major challenges in international health. International Health, 2, 181-185.

Global Ministerial Forum on Research for Health. (2008). Bamako Call to Action on Research for Health, Bamako, Mali.

Gostin, L.O. (2014). Global health law. Cambridge, MA: Harvard University Press.

Hruschka, D.L., Schwartz, D., St. John, D.C., Picone-Decard, E., Jenkins, R.A., and Carey, J.W. (2004). Reliability in coding open ended data: Lesson learned from HIV behavioral research. Field Methods, 16(3), 307-331.

Lairumbi, G.M., Molyneux, S., Snow, R.W., Marsh, K., Peshu, N., and English, M. (2008). Promoting the social value of research in Kenya: Examining the practical aspects of collaborative partnerships using an ethical framework. Social Science and Medicine, 67(5), 734-747.

Ministerial Summit on Health Research. (2004). The Mexico Statement on Health Research, Mexico City.

Anonymous, in press. [details omitted for double-blind reviewing]

Anonymous, et al. (2014). [details omitted for double-blind reviewing]

Powers, M. \& Faden, R. (2006). Social justice: The moral foundations of public health and health policy. Oxford: Oxford University Press. 
Richardson, H. (2002). Democratic autonomy: Public reasoning about the ends of policy. Oxford: Oxford University Press.

Ruger, J.P. (2012). Global health justice and governance. American Journal of Bioethics, 12, 35-54.

Ruger, J.P. (2011). Shared health governance. American Journal of Bioethics, 11, 32-45.

Ruger, J.P. (2008). Normative foundations of global health law. Georgetown Law Journal, 96, 423443.

Shue, H. (1996). Basic rights: Subsistence, affluence, and U.S. foreign policy. Princeton: Princeton University Press.

Young, I.M. (2000). Inclusion and democracy. Oxford: Oxford University Press. 


\section{Figure 1: Ethical guidance linking global health research consortia governance to health}

equity

\section{Furthering health justice}

- Consortia research priorities should relate to improving the health of worst-off individuals within LMICs, e.g. diseases that drive their morbidity and mortality or promoting their equal access and financial protection within health systems.

- Consortia should have research translation objectives and strategies that promote the use of research findings in ways that benefit the health of the worst-off in LMICs.

- Consortia research capacity strengthening objectives and strategies should focus on building independent research capacity at the individual and institutional levels for LMIC partners.

- Consortia priorities should be determined through inclusive and deliberative processes, avoiding disproportionate participation of certain roles and demographics.

- Priority-setting should be led by LMIC consortia members, with high-income country consortia members assuming a supportive and facilitative role.

- Priority-setting should, at a minimum, be informed by the views of research users and beneficiaries within partners' countries. Shared decision-making on priorities, especially with disadvantaged groups, is highly desirable.

\section{Shared resources}

- Substantially more consortia resources should be allocated to LMIC partners' research than to high-income country partners' research.

- A greater proportion of high-income country partners' resources should be allocated to assisting LMIC partners conduct research than to their own research in LMICs.

- Consortia resources should be allocated to equity-oriented research, research capacity development, and research translation activities.

\section{Shared responsibility}

- Consortia leaders, partner institution leaders, and researchers should carry out their specific role-related responsibilities to ensure the consortia conducts research that promotes health equity within LMICs and undertakes research capacity strengthening for LMIC partners.

\section{Mutual collective accountability}

- All consortia members should mutually understand and agree upon equity-oriented outcomes and outputs (for research, research capacity development, and research translation) that the consortia aims to achieve.

- Monitoring and evaluation on these outcomes and outputs should be performed by all consortia partners. 
Table 1: Analysis of FHS annual meeting attendance

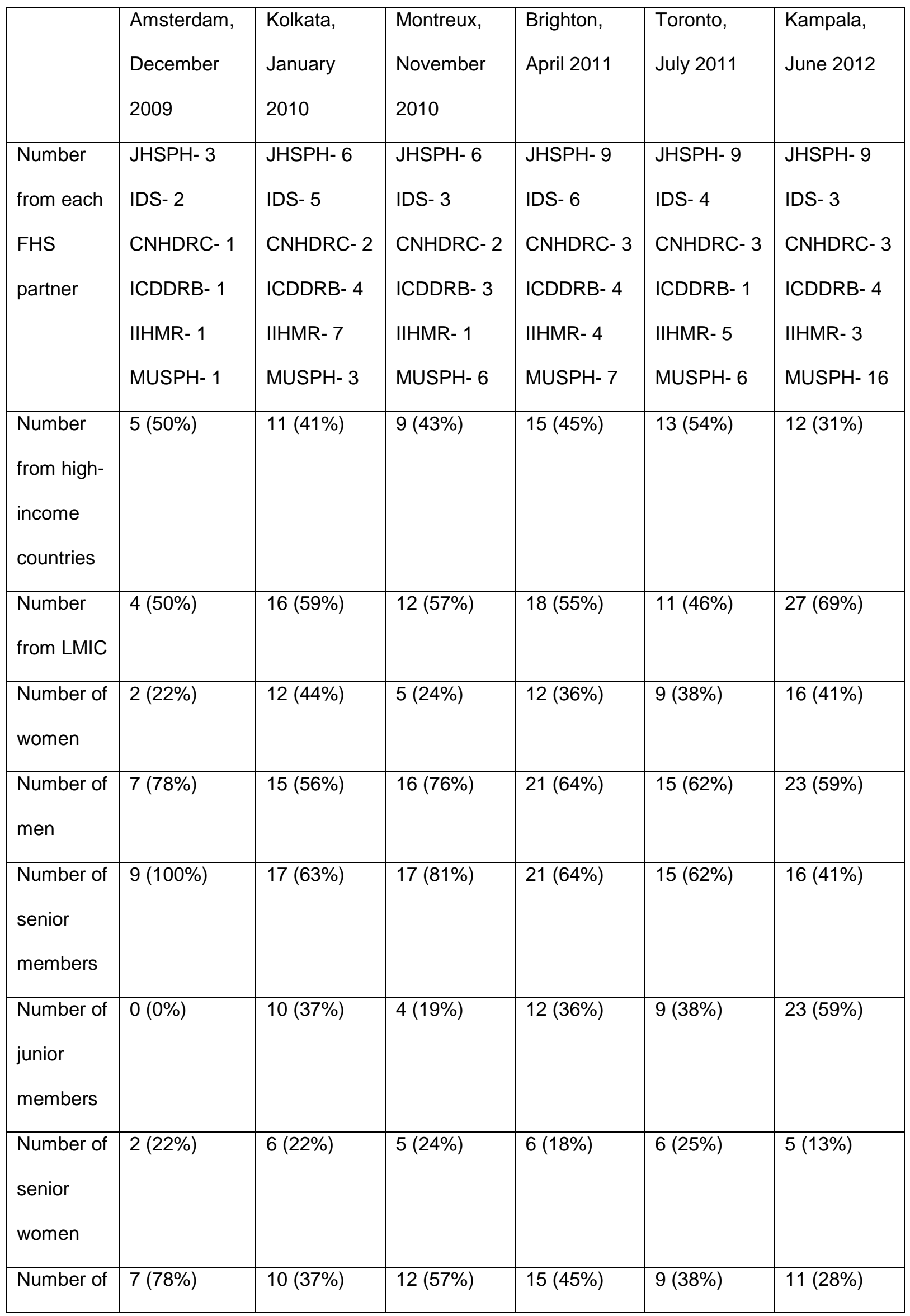




\begin{tabular}{|l|l|l|l|l|l|l|}
\hline senior & & & & & & \\
men & & & & & & \\
\hline
\end{tabular}

Note: Steering committee members and senior faculty (professors, associate professors) were classified as senior consortium members. All other participants were classified as junior consortium members. 
Table 2: Factors facilitating alignment with ethical guidance for shared sovereignty

\begin{tabular}{|c|c|}
\hline Feature & Facilitating factor \\
\hline \multirow[t]{5}{*}{ LMIC-led priority-setting } & Decentralised consortia governance \\
\hline & $\begin{array}{l}\text { DFID funding scheme required demand-driven } \\
\text { research and that at least three consortia } \\
\text { partners be from LMICs }\end{array}$ \\
\hline & LMIC control of budget allocations \\
\hline & Strong research capacity of LMIC partners \\
\hline & $\begin{array}{l}\text { Trust between consortium partners built over } \\
\text { FHS Phase-1 }\end{array}$ \\
\hline Engagement with LMIC stakeholders & DFID funding scheme requirement \\
\hline \multirow[t]{5}{*}{$\begin{array}{l}\text { Equal opportunity to participate and achieving a } \\
\text { deliberative priority-setting process }\end{array}$} & $\begin{array}{l}\text { Having face-to-face meetings; DFID funding } \\
\text { supports annual meetings }\end{array}$ \\
\hline & $\begin{array}{l}\text { DFID funding scheme required a yearlong } \\
\text { Inception Phase }\end{array}$ \\
\hline & $\begin{array}{l}\text { Research capacity strengthening for junior } \\
\text { researchers }\end{array}$ \\
\hline & $\begin{array}{l}\text { Female CEO and strong female voices in } \\
\text { consortium }\end{array}$ \\
\hline & $\begin{array}{l}\text { Trust between consortium partners built over } \\
\text { FHS Phase-1 }\end{array}$ \\
\hline
\end{tabular}


Table 3: Factors that constrained alignment with ethical guidance for shared sovereignty

\begin{tabular}{|c|c|}
\hline Feature & Challenge \\
\hline LMIC-led priority-setting & $\begin{array}{l}\text { Funding environment: Competitiveness of grant } \\
\text { application was enhanced if JHSPH was the } \\
\text { administering institution due to its strong financial } \\
\text { management track record with large grants }\end{array}$ \\
\hline Inclusion of consortia members & Cost: Not everyone could attend annual meetings \\
\hline \multirow{3}{*}{$\begin{array}{l}\text { Engagement of LMIC stakeholders during } \\
\text { priority-setting, especially disadvantaged } \\
\text { populations }\end{array}$} & Advice was too generalised to be helpful \\
\hline & $\begin{array}{l}\text { Different types of national governments } \\
\text { sometimes may present a barrier to engaging } \\
\text { civil society and bringing out voices of } \\
\text { disadvantaged to set research priorities }\end{array}$ \\
\hline & $\begin{array}{l}\text { DFID funding scheme emphasised policymaker } \\
\text { engagement }\end{array}$ \\
\hline
\end{tabular}




\section{University Library}

\section{- M M N E R VA A gateway to Melbourne's research publications}

Minerva Access is the Institutional Repository of The University of Melbourne

Author/s:

Pratt, B;Hyder, AA

Title:

Governance of global health research consortia: Sharing sovereignty and resources within Future Health Systems

Date:

2017-02-01

\section{Citation:}

Pratt, B. \& Hyder, A. A. (2017). Governance of global health research consortia: Sharing sovereignty and resources within Future Health Systems. SOCIAL SCIENCE \& MEDICINE, 174, pp.113-121. https://doi.org/10.1016/j.socscimed.2016.11.039.

Persistent Link:

http://hdl.handle.net/11343/123351 